\title{
IL-10 modulates DSS-induced colitis through a macrophage-ROS-NO axis
}

\author{
B Li, R Alli, P Vogel and TL Geiger
}

Breakdown of the epithelial barrier because of toxins or other insults leads to severe colitis. Interleukin-10 (IL-10) is a critical regulator of this, yet its cellular targets and mechanisms of action are not resolved. We address this here. Mice with a macrophage-selective deletion of IL-10R $\alpha$ (IL-10R $\alpha^{\text {Mdel }}$ ) developed markedly enhanced dextran sodium sulfate (DSS)-induced colitis that did not significantly differ from disease in IL-10 ${ }^{-1-}$ or IL-10R $\alpha^{-1-}$ mice; no impact of IL-10R $\alpha$ deficiency in other lineages was observed. IL-10R $\alpha^{\text {Mdel }}$ colitis was associated with increased mucosal barrier disruption in the setting of intact epithelial regeneration. Lamina propria macrophages (LPM $\phi s)$ did not show numerical or phenotypic differences from controls, or a competitive advantage over wild-type cells. Proinflammatory cytokine production, and particularly tumor necrosis factor- $\alpha$ (TNF- $\alpha$ ), was increased, although TNF- $\alpha$ neutralization failed to reveal a defining role for this cytokine in the aggravated disease. Rather, IL-10R $\alpha^{\text {Mdel }}$ LPM $\phi s$ produced substantially greater levels of nitric oxide (NO) and reactive oxygen species (ROS) than controls. Inhibition of these had modest effects in wild-type mice, although they dramatically reduced colitis severity in IL-10R $\alpha^{\text {Mdel }}$ mice, and largely eliminated the differential effect of DSS in them. Therefore, the palliative actions of IL-10 in DSS-induced colitis predominantly results from its macrophage-specific effects. Downregulation of NO and ROS production are central to the protective actions of IL-10.

\section{INTRODUCTION}

Inflammatory bowel diseases (IBD), including ulcerative colitis and Crohn's disease, are characterized by mucosal damage and ulceration. Breech of the intestinal epithelial barrier by commensal bacteria triggers the inflammation that is responsible for IBD pathogenesis. Dextran sodium sulfate (DSS) administration has commonly been used to model ulcerative colitis. Ingested DSS concentrates in the colon where it disrupts the epithelial barrier and induces a secondary inflammatory response characterized by the production of proinflammatory cytokines, including interleukin (IL)-1 $\beta$, IL-6, IL-12, IL-18, and tumor necrosis factor- $\alpha$ (TNF- $\alpha) .{ }^{1-6}$

IL-10 is a predominantly anti-inflammatory cytokine with an essential role in maintaining gastrointestinal homeostasis. Genetic variants in IL-10 or the IL-10 receptor (IL-10R) are associated with IBD susceptibility. Older IL- $10^{-1-}$ mice develop spontaneous colitis, and IL-10 deficiency exacerbates colitis in several models, including DSS and T-cell transfer colitis. $^{7-11}$ Moreover, pharmacologically administered IL-10 ameliorates colitis in mice by inhibiting intestinal inflammation and suppressing proinflammatory cytokine production. ${ }^{12-14}$ IL-10 is produced by hematopoietically derived cells, including $\mathrm{T}$ cells, B cells, dendritic cells (DCs), and macrophages. Signaling through the IL-10R downmodulates TNF- $\alpha$ production and proinflammatory signaling through various mechanisms, including the induction of SOCS3, other antiinflammatory proteins, and miRNA. ${ }^{15-17}$

The cell types responsible for the anti-inflammatory effects of IL-10 and mucosal protection in colitis have not been resolved. The IL-10R is a heterodimer comprising an IL-10R $\alpha$ chain that is specific for IL-10 and an IL-10R $\beta$ that is shared with other IL-10-family cytokines, including IL-22, IL-26, and the interferon- $\lambda$ family. Whereas IL-10R $\beta$ is largely restricted to hematopoietic cells, IL-10R $\alpha$ is broadly expressed. We recently described the production of mice allowing the conditional deletion of IL-10R $\alpha$ that we apply here to assess how lineagespecific IL-10 responsiveness influences colitis severity. ${ }^{18} \mathrm{We}$ identify a selective role for macrophage IL-10R $\alpha$, and find suppression of nitric oxide (NO) and reactive oxygen species (ROS) production to be critical downstream mechanisms.

Department of Pathology, St Jude Children's Research Hospital, Memphis, Tennessee, USA. Correspondence: TL Geiger (terrence.geiger@stjude.org) Received 17 July 2013; accepted 27 October 2013; published online 4 December 2013. doi:10.1038/mi.2013.103 


\section{RESULTS \\ Macrophage IL-10 response limits the severity of DSS-induced colitis}

To define the cellular lineages responsible for the protective effect of IL-10 in colitis, we first generated mixed chimeras in which bone marrow from mice with a germline deletion of IL-10R $\alpha\left(\mathrm{CMV}-\mathrm{Cre} \times \mathrm{IL}-10 \mathrm{R} \alpha^{\mathrm{fl} / \mathrm{fl}}\right.$; IL-10R $\left.\alpha^{-/-}\right)$or wild-type (WT) controls was transplanted in a crisscross manner into lethally irradiated IL-10R $\alpha^{-/-}$or IL-10R $\alpha^{\mathrm{WT}}$ recipients. Colitis was induced by oral administration of $3 \%$ DSS for 5 days. No effect of IL-10R $\alpha$ deficiency restricted to radioresistant host populations was seen (Supplementary Figure S1 online). In contrast, mice with IL-10R $\alpha$ deficiency in the bone marrow grafts developed significantly worse disease compared with those receiving WT bone marrow. This implies that radiosensitive hematopoietic populations are primarily responsible for IL-10's effects.

To further dissect the lineages responsible, we bred C57BL/ 6 -IL-10R $\alpha^{\mathrm{fl} / \mathrm{fl}}$ mice with mice expressing Cre transgenes in macrophages (Lys-Cre; IL-10R $\alpha^{\text {Mdel }}$ ), T cells (CD4-Cre; IL-10R $\left.\alpha^{\text {Tdel }}\right)$, DCs (CD11c-Cre; IL-10R $\alpha^{\text {DCdel }}$ ), or B cells (CD19-Cre; IL-10R $\alpha^{\text {Bdel }}$ ). Cells from the different lines demonstrated an anticipated absence of IL-10R $\alpha$ on Cre-expressing lineages (Supplementary Figure S2 and ref. 18).

After colitis induction, control IL-10R $\alpha^{\mathrm{fl} / \mathrm{fl}}$ mice typically lost $\sim 15-20 \%$ of body weight by days 7 to 8 , with subsequent restoration of initial weight (Figure 1a-d). IL-10R $\alpha^{\text {DCdel, }}$ IL- $10 \mathrm{R} \alpha^{\mathrm{Bdel}}$, and IL-10R $\alpha^{\text {Tdel }}$ mice displayed identical disease kinetics and magnitude, indicating that T-cell, B-cell, and DC responsiveness to IL-10 did not affect clinical severity (Figure 1a-c). In contrast, IL-10R $\alpha^{\mathrm{Mdel}}$ mice developed more severe disease (Figure 1d). Mean maximal weight loss was greater than for controls ( $25 \pm 5$ vs. $16 \pm 4 \%$ ) and $2 / 10$ IL-10R $\alpha^{\text {Mdel }}$ mice but no controls had to be culled because of their illness. At a higher dose of 4\% DSS, 6/10 (60\%) IL-10R $\alpha^{\text {Mdel }}$ mice, although no control mice, died or required killing (Figure 1e).

The Lys-Cre transgene is expressed in granulocytic cells in addition to macrophages. ${ }^{19}$ Comparison of DSS-treated IL- $10 \mathrm{R} \alpha^{\mathrm{fl} / \mathrm{fl}}$ and IL-10R $\alpha^{\mathrm{Mdel}}$ mice indicated that the IL-10R deficiency did not lead to differences in the percent or absolute numbers of granulocytes in the lamina propria (LP; Supplementary Figure S3). To more definitively exclude a role for these cells in the enhanced disease in IL-10R $\alpha^{\text {Mdel }}$ mice, we depleted them with anti-Ly6G antibody (Ab) before DSS administration. ${ }^{20}$ Neutrophils remained undetectable for $>9$ days (data not shown). Despite this, the anti-Ly-6G Ab treatment did not significantly alter disease course or severity in either IL- $10 \mathrm{R} \alpha^{\mathrm{fl} / \mathrm{fl}}$ or IL-10R $\alpha^{\text {Mdel }}$ mice (Figure 1f; $P>0.05$ ). This indicates that macrophage and not granulocytes are responsible for the protective effects of IL-10 in colitis.

To better delineate the contribution of macrophage, we compared disease in IL-10R $\alpha^{\mathrm{Mdel}}$, IL- $10^{-/-}$, and IL-10R $\alpha^{-/-}$ mice. The latter two lines have global deficiency of IL-10 or its specific receptor. All three lines developed more severe disease than IL-10R $\alpha^{\mathrm{fl} / \mathrm{fl}}$ controls (Figure 1g). However, disease in IL-10R $\alpha^{\text {Mdel }}$ mice did not significantly differ at any time point from that in IL-10R $\alpha^{-/-}$or IL-10 $10^{-/-}$mice. Therefore, colitis in IL-10R $\alpha^{\text {Mdel }}$ mice is comparable to that in mice ubiquitously deficient in the IL-10 response. Cumulatively, these results implicate the macrophage lineage as the primary mediator of IL-10 effects in colitis induced by barrier disruption.

\section{Increased immunopathology in IL-10R $\alpha^{\text {Mdel }}$ colonic mucosa}

We anticipated that the enhanced disease in IL-10R $\alpha^{\mathrm{Mdel}}$ mice would be associated with increased mucosal damage and hence gastrointestinal blood loss. Indeed, significantly increased bleeding was seen in IL- $10 \mathrm{R} \alpha^{\mathrm{Mdel}}$ compared with IL- $10 \mathrm{R} \alpha^{\mathrm{fl} / \mathrm{fl}}$ cohorts on each day that blood was detectable (Figure 2a).

This was correlated with histologic changes. IL-10 $\alpha^{\text {Mdel }}$ colons, isolated at day 7 , showed a more extensive cellular infiltrate, increased submucosal edema, and increased epithelial erosion compared with IL-10R $\alpha^{\mathrm{fl} / \mathrm{ll}}$ controls (Figure 2b). Observer-blinded scoring demonstrated a significantly greater area of tissue destruction and severity of inflammation, ulceration, and hyperplasia in the IL-10 $\mathrm{R} \alpha^{\mathrm{Mdel}}$ colons. Total histologic score was $2.7 \pm 2.3$ and $7.6 \pm 1.8$ (scale $0-12$ ) for IL-10R $\alpha^{\mathrm{fl} / \mathrm{fl}}$ and IL-10R $\alpha^{\mathrm{Mdel}}$ mice, respectively (Figure 2c). Consistently, IL-10R $\alpha^{\mathrm{Mdel}}$ colons were shorter than controls $(5.8 \pm 0.6$ vs. $7.2 \pm 0.8 \mathrm{~cm}$, Figure $2 \mathrm{~d}, \mathrm{e})$. Therefore, by clinical, gross pathologic, and histopathologic measures, IL-10R $\alpha^{\text {Mdel }}$ mice develop colitis that is increased in severity.

Importantly, we did not observe clinical evidence of spontaneous colitis in our IL-10R $\alpha^{\mathrm{Mdel}}$ colony, which was housed under Helicobacter spp.-free conditions, arguing against the development of subclinical disease before DSS administration. We verified this histologically. Colon tissue sections from unmanipulated IL-10R $\alpha^{\mathrm{fl} / \mathrm{fl}}$ and IL-10R $\alpha^{\mathrm{Mdel}}$ mice were equivalent, and abnormalities indicating incipient colitis were not observed (data not shown).

\section{Unimpaired epithelial regeneration in IL-10R $\alpha^{\text {Mdel }}$ mice}

To assess for alterations in barrier integrity with loss of macrophage IL-10 responsiveness, we administered fluorescein isothiocyanate (FITC)-dextran by gastric lavage and measured its passage into the blood stream. Levels of FITC-dextran were greater in IL-10R $\alpha^{\text {Mdel }}$ than IL-10R $\alpha^{\text {fl/fl }}$ blood (Supplementary Figure S4a), consistent with increased barrier disruption.

Impaired epithelial regeneration from crypt progenitors has been associated with enhanced colitic inflammation, ${ }^{21}$ and may have contributed to the barrier disruption. We analyzed this by pulsing unmanipulated or colitic IL-10R $\alpha^{\text {Mdel }}$ or IL-10R $\alpha^{\mathrm{fl} / \mathrm{fl}}$ mice with 5 -bromodeoxyuridine for $2 \mathrm{~h}$, and then measuring its incorporation into the colonic epithelium. No significant difference was detected between IL-10R $\alpha^{\text {Mdel }}$ and IL-10R $\alpha^{\mathrm{fl} / \mathrm{fl}}$ colons, either in untreated mice or mice receiving DSS (Supplementary Figure S4b), indicating that differential epithelial turnover is not responsible for the different disease susceptibilities. 

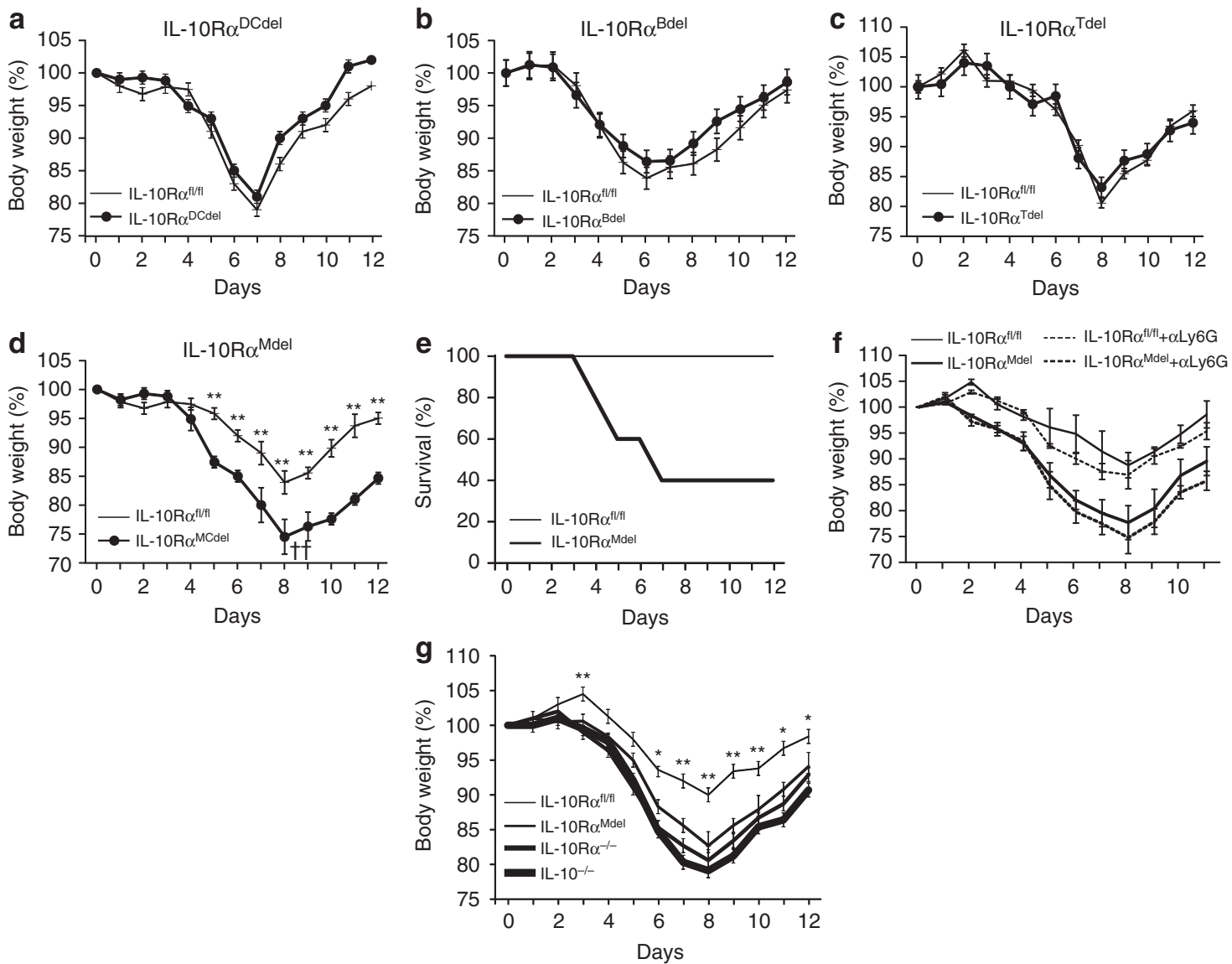

Figure 1 Macrophage interleukin (IL)-10R $\alpha$ expression protects mice from dextran sodium sulfate (DSS)-induced colitis. (a) IL-10R $\alpha^{\mathrm{DCdel}}$ (b) IL-10R $\alpha^{\text {Bdel }}$, (c) IL-10R $\alpha^{\text {Tdel }}$, and (d) IL-10R $\alpha^{\text {Mdel }}$ mice or littermate Cre ${ }^{-}$(IL-10R $\left.\alpha^{\text {fl/fll }}\right)$ controls $(n=10 /$ cohort) received $3 \%$ DSS solution in drinking water ad libitum for 5 days. Mean \pm s.e.m. percent of initial body weight is plotted. ${ }^{* *} P<0.01 ;^{\dagger}$ death event. (e) IL-10R $\alpha^{\mathrm{Mdel}}$ and IL-10R $\alpha^{\mathrm{f} / \mathrm{fl}}$ mice ( $n=10 /$ cohort) were treated with $4 \%$ DSS for 5 days and survival monitored. (f) IL-10R $\alpha^{\text {Mdel }}$ mice and IL-10R $\alpha^{\mathrm{fl} / \mathrm{fl}}$ controls $(n=10 / \mathrm{cohort})$ were depleted of neutrophils with $1 \mathrm{~A} 8$ antibody or received control rat immunoglobulin $\mathrm{G}(\mathrm{IgG}) 1$ day before $3 \%$ DSS administration. Data are representative of three independent experiments. (g) IL-10 ${ }^{-1-}, \mathrm{IL}-10 \mathrm{R} \alpha^{-1-}$, and IL-10R $\alpha^{\mathrm{fl} / \mathrm{fl}}$ mice were treated with $3 \% \mathrm{DSS}$ for 5 days and body weight monitored. Data are representative of three independent experiments. ${ }^{\star} P<0.05$, ${ }^{\star *} P<0.01$ for IL-10R $\alpha^{\text {Mdel }}$ vs. IL-10R $\alpha^{\text {fl/fl }}$. Significant differences between IL-10R $\alpha^{\mathrm{Mdel}}$, IL-10R $\alpha^{-1-}$, and IL-10 $10^{-1-}$ cohorts are not seen.

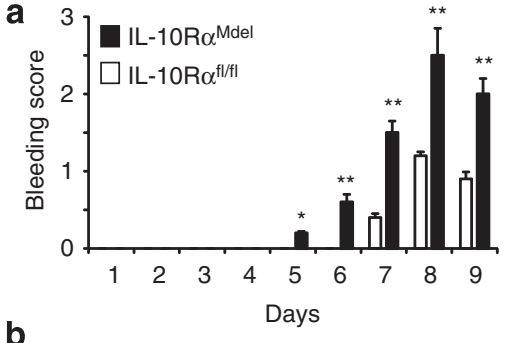

b

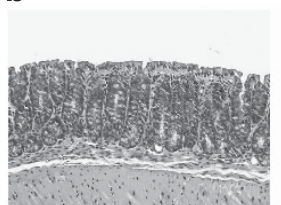

Untreated

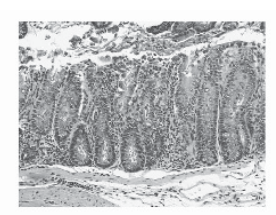

$\mathrm{IL}-10 \mathrm{R} \alpha^{\mathrm{f} / \mathrm{fl}}$
C Total score
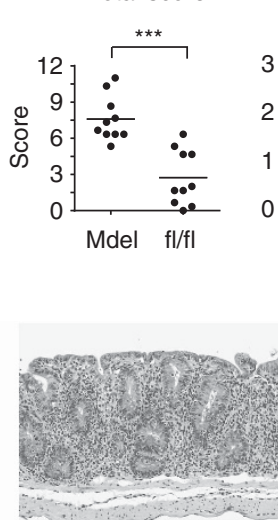

IL-10R $\alpha^{\text {Mdel }}$

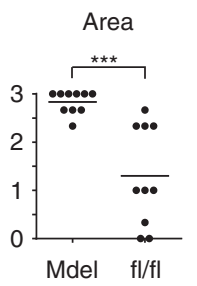

d
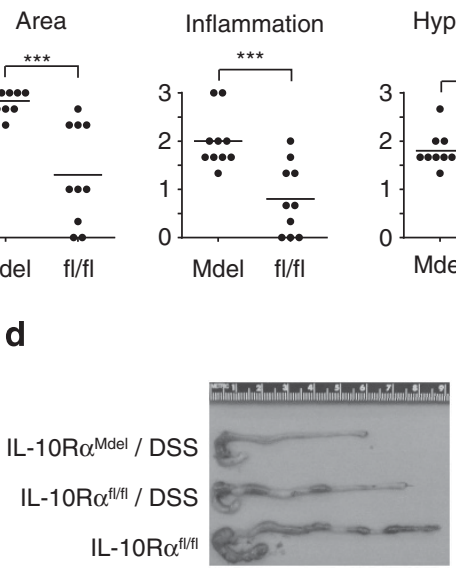

Hyperplasia

Ulceration
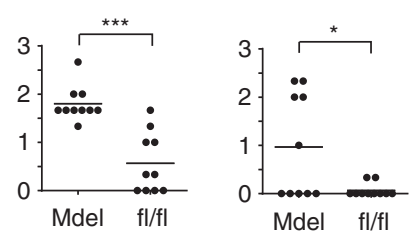

e

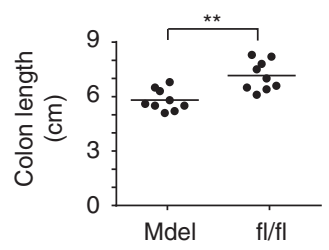

Figure 2 Dextran sodium sulfate (DSS)-induced colitis in interleukin (IL)-10R $\alpha^{\text {Mdel }}$ mice. (a) IL-10R $\alpha^{\text {Mdel }}$ and IL-10R $\alpha^{\text {fl/fl }}$ mice received $3 \%$ DSS for 5 days, and rectal bleeding was scored daily. (b, c) Representative photomicrographs and tallied scores for disease parameters from hematoxylin and eosin (H\&E)-stained colon sections obtained 7 days after initiating DSS treatment. Scoring for individual parameters is scaled from 0 to 3 (0-12 total) and criteria are listed under Methods. Mean values for individual mice (circles) and cohorts (lines) are plotted. (d, e) Colons were removed at day 7 and colon length measured. Individual mice (circles) and cohort means (lines) are plotted. ${ }^{\star} P<0.05$, ${ }^{\star \star} P<0.01,{ }^{\star \star \star} P<0.001$. 


\section{Macrophage infiltrate in DSS colitis}

As an alternative explanation for the enhanced IL-10R $\alpha^{\text {Mdel }}$ colitis, we looked for changes in the number and maturation state of IL-10R $\alpha^{\text {Mdel }}$ lamina propria macrophages (LPM $\phi$ s). Surprisingly, gated colonic $\mathrm{CD}_{11} \mathrm{~b}^{+} \mathrm{F} 4 / 80^{+} \mathrm{Ly}_{6 \mathrm{G}}{ }^{-/ \mathrm{lo}}$ $\mathrm{CD} 11 \mathrm{c}^{-/ \mathrm{dim}} \mathrm{LPM} \phi \mathrm{s}$, a population we also characterized as $\mathrm{CD}_{4} 5^{+}$and $\mathrm{SiglecF}^{-}$, were not significantly increased in colitic IL-10R $\alpha^{\text {Mdel }}$ compared with IL-10R $\alpha^{\mathrm{fl} / \mathrm{fl}}$ mice (Figure 3a and Supplementary Figure S5).

LPM $\phi$ s are functionally diverse. Takada et al. $^{22}$ separated $\mathrm{CD}_{11 \mathrm{~b}}{ }^{+} \mathrm{F} 4 / 80^{+} \mathrm{CD}_{11 \mathrm{c}^{-} \mathrm{LPM} \phi \text { s into a SSC }}{ }^{\text {hi }}$ population, referred to as $\operatorname{LPM} \phi 1$, and a SSC ${ }^{\text {lo }}$ population, $\operatorname{LPM} \phi 2$, with distinct cytokine production and chemokine response properties. LPM $\phi$ subsets expressing CD11c have been more recently identified during intestinal inflammation. ${ }^{23}$ We assessed LPM $\phi$ s, gated to include $\mathrm{CD} 11 \mathrm{c}^{-}$and CD11c dim cells, in IL- $10 \mathrm{R} \alpha^{\mathrm{Mdel}}$ and IL-10R $\alpha^{\mathrm{fl} / \mathrm{fl}}$ mice with colitis. These did segregate into discrete $\mathrm{SSC}^{\mathrm{hi}}$ and $\mathrm{SSC}^{\text {lo }}$ populations (Figure $\mathbf{3 b}$ ). However, the proportions of $\mathrm{SSC}^{\mathrm{hi}}$ and $\mathrm{SSC}^{\mathrm{lo}}$ cells did not significantly differ (Figure 3c). Furthermore, markers associated with LPM $\phi$ activation and subset assignment, including CD40, CD80, CD86, and Toll-like receptor 2, were comparably expressed in IL-10R $\alpha^{\text {Mdel }}$ and IL-10R $\alpha^{\mathrm{fl} / \mathrm{fl}} \mathrm{LPM} \phi \mathrm{s}$ (Supplementary Figure S6). Therefore, despite the difference in disease severity, IL-10R $\alpha^{\text {Mdel }}$ and IL-10R $\alpha^{\mathrm{fl} / \mathrm{fl}} \mathrm{LPM} \phi \mathrm{s}$ are phenotypically similar and present in similar numbers.

\section{IL-10R $\alpha^{\text {Mdel }}$ macrophages actively promote colitis and do not outcompete WT cells}

To gain insight into whether IL-10R $\alpha^{\mathrm{Mdel}}$ macrophages play a dominant role in increasing colitis severity, we generated hematopoietic chimeras in which lethally irradiated WT recipients received stem cell rescue with WT, IL-10R $\alpha^{\mathrm{Mdel}}$, or a mixture of WT and IL-10R $\alpha^{\text {Mdel }}$ bone marrow. Cell origins were distinguishable by the alternative expression of CD45.1 and CD45.2, allowing us to compare the populations in a competitive manner. As anticipated, recipients of IL-10R $\alpha^{\text {Mdel }}$ marrow developed more severe colitis than those receiving WT marrow. However, mice receiving a mixture of WT and IL-10R $\alpha^{\mathrm{Mdel}}$ marrow developed disease essentially identical to those receiving IL-10R $\alpha^{\text {Mdel }}$ marrow alone (Supplementary Figure S7) despite equivalent proportions of IL-10R $\alpha^{\text {Mdel }}$ and IL-10R $\alpha^{\mathrm{fl} / \mathrm{fl}}$ cells among transferred bone marrow cells, blood macrophage before colitis induction, and macrophage in the LP, spleen, and blood in diseased mice (data not shown). Implicitly, altered macrophage function rather than competitiveness acts to worsen disease in IL- $10 \mathrm{R} \alpha^{\mathrm{Mdel}}$ mice.

\section{Increased proinflammatory cytokine production in IL-10R $\alpha^{\text {Mdel }}$ colons}

To functionally analyze the impact of the IL-10R $\alpha^{\text {Mdel }}$ mutation, we next measured levels of IL-1 $\beta$, IL-18, IL-6, monocyte chemotactic protein- 1 , and TNF- $\alpha$, proinflammatory cytokines associated with colitis, in whole colons from day 7 colitic mice. Each was increased in IL-10R $\alpha^{\text {Mdel }}$ compared with IL-10R $\alpha^{\mathrm{fl} / \mathrm{fl}}$ controls (Figure $4 \mathrm{a}, P<0.05$ ). IL-10 is also produced by activated macrophages, initiating an autocrine-
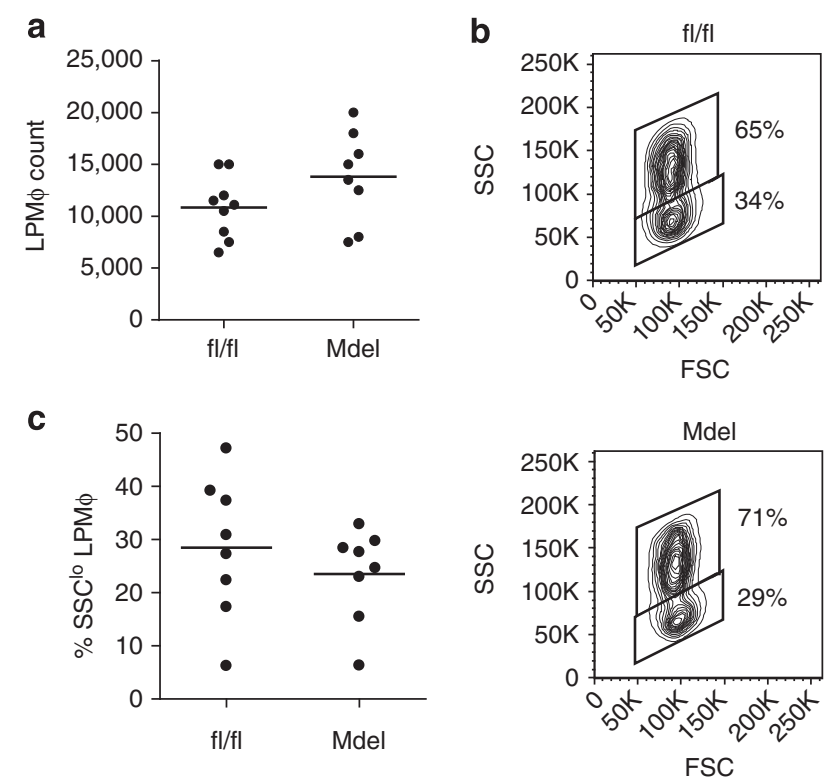

Figure 3 Analysis of lamina propria macrophages (LPM $\phi$ s). (a) Cells were isolated from large intestine lamina propria of IL-10R $\alpha$ Mdel and $\mathrm{IL}-10 \mathrm{R} \alpha^{\mathrm{fl} / \mathrm{fl}}$ mice on day 7 after colitis induction. Absolute numbers of

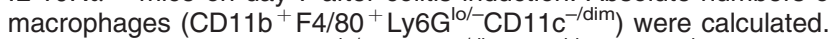
$(\mathbf{b}, \mathbf{c}) \mathrm{CD} 11 \mathrm{~b}^{+} \mathrm{F} 4 / 80^{+} \mathrm{Ly}_{6 \mathrm{G}^{\mathrm{lo} /}-} \mathrm{CD} 11 \mathrm{c}^{-/ \mathrm{dim}} \mathrm{SSC}^{\mathrm{hi}}$ and SSC $\mathrm{SO}^{\mathrm{lo}} \mathrm{LPM} \phi \mathrm{s}$ were distinguished by flow cytometry (b). The proportion of $\mathrm{SSC}^{\text {lo }}$ cells among total $\left(\mathrm{SSC}^{\text {hi }}+\mathrm{SSC}^{\mathrm{lo}}\right) \mathrm{LPM} \phi \mathrm{S}$ is plotted (c). Results from individual mice (circles) and population means (lines) are plotted. Differences are not significant. FSC, forward scatter; SSC, side scatter.

and paracrine-negative feedback loop. However, IL-10 levels were unaffected, indicating that the inability of macrophages to respond to IL-10 did not influence its overall quantity.

We further characterized specific cytokine production by macrophages themselves. Cytokine transcription was measured in neutrophil-depleted flow-sorted $\mathrm{CD}_{11 \mathrm{~b}}{ }^{+} \mathrm{F} 4 / 80^{+}$ CD11c ${ }^{-/ \text {dim }_{\text {Ly6G }}}{ }^{-/ \mathrm{lo}}$ LPM $\phi$ s. A $4.4 \pm 0.6-, 2.8 \pm 0.4-$, and $1.5 \pm 0.2$-fold increase in IL- $1 \beta$, TNF- $\alpha$, and IL-12p35 message, respectively, was seen in IL-10R $\alpha^{\text {Mdel }}$ compared with IL-10R $\alpha^{\mathrm{fl} / \mathrm{fl}}$ LPM $\phi$ s (Figure 4b). In contrast, transforming growth factor- $\beta$ message was decreased in IL- $10 \mathrm{R} \alpha^{\text {Mdel }}$ LPM $\phi$ s by $0.42 \pm 0.08$-fold, whereas IL-6, IL-10, and IL-23p19 mRNA were essentially unchanged. Therefore, loss of macrophage responsiveness to IL-10 leads to an overall shift toward increased proinflammatory cytokine production.

The role of T helper type 17 cells in DSS colitis is unclear, with one study indicating positive and negatives roles for IL-17F and IL-17A, respectively, and another identifying disease-promoting effects of IL-17A. ${ }^{4,24}$ We did not observe differences in IL17A levels in whole colons or in the numbers of IL-17A- or IL17F-positive infiltrating T lymphocytes during DSS colitis when comparing IL-10R $\alpha^{\mathrm{Mdel}}$ and IL-10R $\alpha^{\mathrm{fl} / \mathrm{fl}}$ mice (data not shown). Considering this and the similar IL-23 mRNA expression in IL$10 \mathrm{R} \alpha^{\mathrm{Mdel}}$ and IL-10R $\alpha^{\mathrm{fl} / \mathrm{fl}}$ LPM $\phi$ s, modulation of Th17 cells does not appear to play a role in the differential disease.

TNF- $\alpha$ is among the earliest macrophage biomarkers produced in DSS colitis. It promotes secondary secretion of other proinflammatory cytokines, and is potently downmodulated by IL-10. ${ }^{25}$ These features, together with the increased 
a
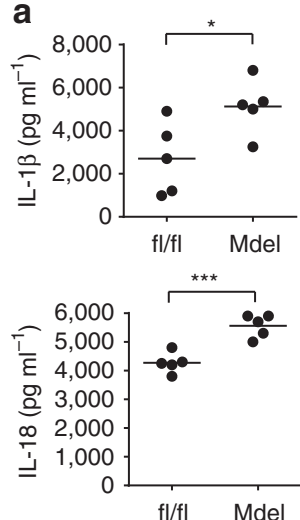
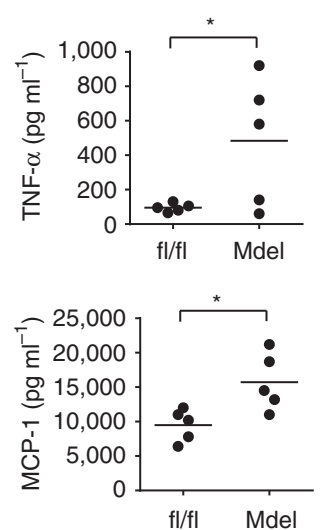
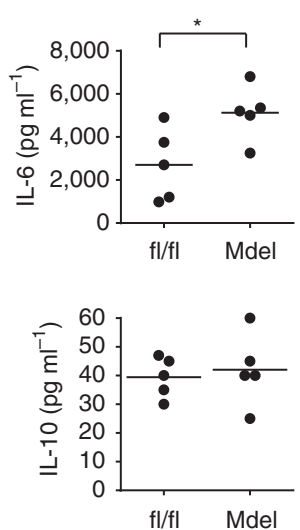

b

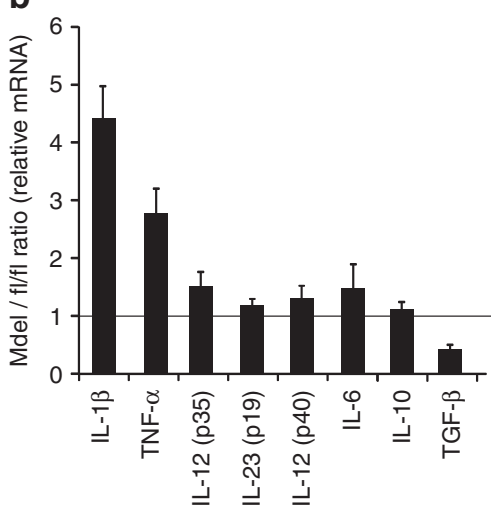

Figure 4 Inflammatory cytokine production by IL-10R $\alpha$-deficient macrophages. (a) Colons from IL-10R $\alpha^{\mathrm{Mdel}}$ and IL-10R $\alpha^{\mathrm{fl} / \mathrm{fl}}$ mice, at 7 days after colitis induction, were homogenized and cytokine content measured by ELISA or multiplex assay. Results from individual mice (circles) and cohort means (lines) are plotted. (b) Relative expression levels (mean \pm 1 s.d.) of the indicated mRNAs from macrophages sorted from colon tissue was measured by qRT-PCR. ${ }^{\star} P<0.05,{ }^{\star \star \star} P<0.001$. Data are representative of three independent experiments. IL, interleukin; MCP-1, monocyte chemotactic protein-1; qRT-PCR, quantitative real-time reverse-transcriptase-PCR; TGF- $\beta$, transforming growth factor- $\beta$; TNF- $\alpha$, tumor necrosis factor- $\alpha$.

production of TNF- $\alpha$ in IL-10R $\alpha^{\text {Mdel }}$ colons, potentially implicate it in the exacerbated disease. To test this, we blocked its activity using anti-TNF- $\alpha$ Ab. Treatment reduced disease severity in both IL- $10 \mathrm{R} \alpha^{\mathrm{fl} / \mathrm{fl}}$ and IL-10R $\alpha^{\mathrm{Mdel}}$ mice (Supplementary Figure S8). However, the extent of this was similar in each line, and treated IL-10R $\alpha^{\text {Mdel }}$ mice still developed more severe disease than even untreated IL-10R $\alpha^{\mathrm{fl} / \mathrm{fl}}$ controls $(P<0.01)$. Therefore, increased TNF- $\alpha$ production may play a role but is inadequate in itself to explain the heightened IL-10R $\alpha^{\text {Mdel }}$ disease.

\section{NO modulation of IL-10R $\alpha^{\text {Mdel }}$ colitis}

IL-10 potently inhibits inducible nitric oxide synthase (iNOS), and thereby NO production. The integrated effects of NO antimicrobial activity, toxic actions on the barrier, and cell signaling activity may alternatively promote or diminish colitis. In DSS colitis, excessive NO production worsens disease, although protective effects of NO have also been identified. ${ }^{26}$ To assess iNOS activity, we sorted LPM $\phi$ s from colitic mice and quantified iNOS mRNA. Levels were $4.7 \pm 0.8$-fold higher in IL-10R $\alpha^{\text {Mdel }}$ compared with IL-10R $\alpha^{\mathrm{fl} / \mathrm{fl}}$ macrophages. Arginase, which inhibits iNOS by degrading NO's nitrogen source, was reciprocally, although less strongly, decreased $(0.52 \pm 0.07$-fold, Figure 5a).

We also analyzed the accumulation of iNOS in homogenized LP cells from colitic mice using an assay for iNOS functional activity. This indicated a $>2$-fold increased activity in IL-10R $\alpha^{\text {Mdel }}$ than IL-10R $\alpha^{\text {fl/fl }}$ colons (Figure $5 \mathbf{b}, 10.8 \pm 3.3$ vs. $5.2 \pm 2.2 \mu \mathrm{mol}$ nitrite produced per $\mu \mathrm{g}$ protein).

To assess the impact of this NO, cohorts of IL-10R $\alpha^{\text {Mdel }}$ or IL-10R $\alpha^{\mathrm{fl} / \mathrm{fl}}$ mice were treated with aminoguanidine hydrochloride $(\mathrm{AG})$, a selective iNOS inhibitor. Consistent with the mixed roles of NO in DSS colitis, treatment of IL-10R $\alpha^{\text {fl/fl }}$ mice led to only a mild and nonsignificant trend toward reduced disease severity (Figure 5c). In contrast, a more substantial protective effect was apparent in IL-10 R $\alpha^{\mathrm{Mdel}}$ mice. Mean weight loss at disease peak in AG-treated IL-10R $\alpha^{\text {Mdel }}$ mice was $15 \pm 3 \%$ vs. $24 \pm 4 \%$ for untreated mice $(P<0.05)$. A similar pattern was observed when comparing bleeding scores and colon lengths for the different treatments (Figure 5d and Supplementary Figure S9). There was a nonsignificant trend toward diminished bleeding in AG-treated vs. untreated IL-10R $\alpha^{\mathrm{fl} / \mathrm{fl}}$ mice; however, this proved significant and more substantial in IL-10R $\alpha^{\text {Mdel }}$ mice.

As an alternative approach to address the role of $\mathrm{NO}$ production, we selectively inhibited arginase with $S$-(2boronoethyl)-l-cysteine (BEC). BEC increased peak weight loss in IL- $10 \mathrm{R} \alpha^{\mathrm{fl} / f \mathrm{l}}$ mice $(17 \pm 3 \%$ treated vs. $12 \pm 2 \%$ untreated, $P<0.05$, Figure 5e). Furthermore, bleeding scores in $\mathrm{IL}-10 \mathrm{R} \alpha^{\mathrm{fl} / \mathrm{fl}}$ mice were significantly greater in the mice receiving BEC (Figure 5f, $P<0.05$ ) than untreated controls. Therefore, arginase inhibition promotes disease in WT mice. In contrast, BEC did not significantly affect weight loss or bleeding score in IL- $10 \mathrm{R} \alpha^{\mathrm{Mdel}}$ mice, although a nonsignificant trend toward increased bleeding was apparent. Colon length measurements showed similar trends (Supplementary Figure S9 online). Therefore, arginase inhibition, which will increase NO production, preferentially promotes colitis in IL-10R $\alpha^{\mathrm{fl} / \mathrm{fl}}$ mice. In IL-10R $\alpha^{\text {Mdel }}$ mice, where NO production is already elevated and arginase diminished, an effect of further reduction in arginase activity is not detected. Cumulatively, these results indicate a role for elevated NO production in the aggravated colitis in IL-10R $\alpha^{\text {Mdel }}$ mice.

Increased reactive oxygen generation in IL-10R $\alpha^{\text {Mdel }}$ colitis ROS production is regulated by IL-10 and is implicated in colitis. Analysis of $\mathrm{p} 47 \mathrm{phox}^{-1-}$ mice demonstrated no difference from controls in DSS colitis severity. Nevertheless, as for NO, the role of ROS in colitis is multifaceted. ROS is important in mediating protection against bacteria entering the mucosa. ${ }^{27}$ At the same time, excess production may be damaging. Indeed, ROS production defects or antioxidant treatments can potentiate disease protection in some circumstances. $^{26,28}$ 


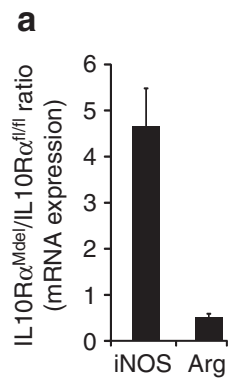

b

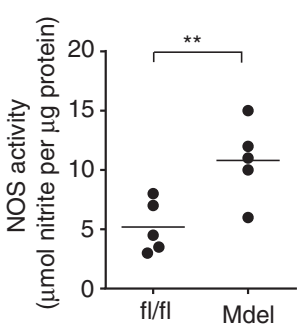

C 110

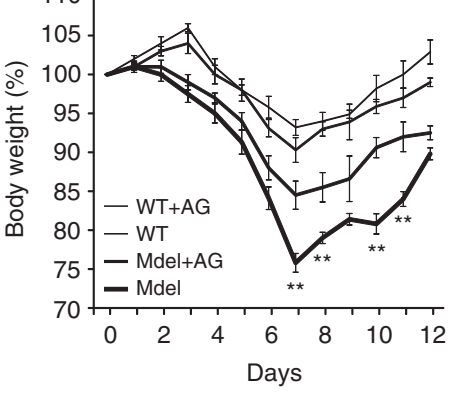

d

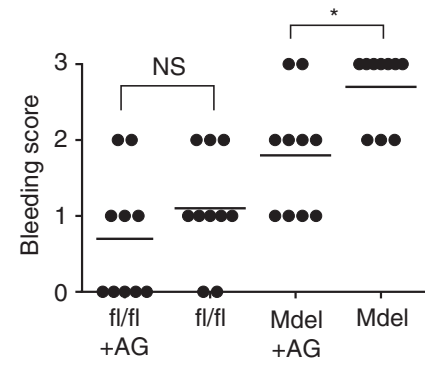

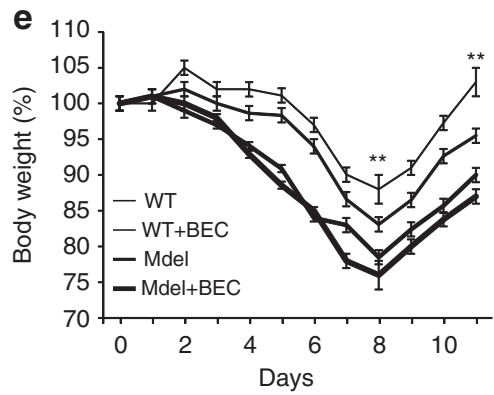

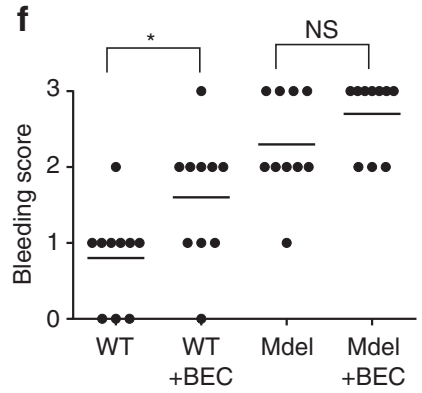

Figure 5 Role of nitric oxide (NO) and arginase in colitis exacerbation. (a) Macrophages were sorted from colons of mice with colitis (day 7) and inducible nitric oxide synthase (iNOS) and arginase expression were measured by quantitative real-time reverse-transcriptase-PCR (qRT-PCR). The ratio of expression in interleukin (IL)-10R $\alpha^{\mathrm{Mdel}}$ to IL-10R $\alpha^{\mathrm{fl} / \mathrm{fl}}$ cells was measured. Mean \pm 1 s.d. is plotted. (b) Colon tissue from mice with colitis (day 7 ) was homogenized and tissue NOS activity measured using a colorimetric assay. Results from individual mice (circles) and cohort means (lines) are shown. (c) Colitis was induced in IL-10R $\alpha^{\text {Mdel }}$ and IL-10R $\alpha^{\text {fl/fl }}$ mice with $3 \%$ dextran sodium sulfate (DSS). Aminoguanidine hydrochloride (AG) or saline was administered intraperitoneally (i.p.). Mean \pm 1 s.e.m. weight change from day 0 is plotted ( $n=10 /$ cohort). (d) Rectal bleeding scores measured on day 7 after colitis induction. (e, f) Analyses are similar to $\mathbf{c}$ and $\mathbf{d}$ except that IL-10R $\alpha^{\mathrm{Mdel}}$ and IL-10R $\alpha^{\mathrm{fl} / \mathrm{fl}}$ mice $\left(n=10 / \mathrm{cohort}^{\mathrm{f}}\right.$ were treated with $S$-(2-boronoethyl)-I-cysteine (BEC) or saline by i.p. injection. ${ }^{\star} P<0.05,{ }^{* *} P<0.01$, NS, not significant. For experiments in c-f, statistical significance is only shown comparing drug-treated and untreated IL-10R $\alpha^{\text {Mdel }}$ or IL-10R $\alpha^{\mathrm{fl} / \mathrm{fl}}$ mice. Significance levels between IL-10R $\alpha^{\mathrm{Mdel}}$ and $\mathrm{IL}-10 \mathrm{R} \alpha \alpha^{\mathrm{fl} / \mathrm{fl}}$ cohorts are not shown. Data are representative of two independent experiments.

We measured ROS production in LPM $\phi$ s by staining with CM-H2DCFDA. ROS was undetectable in LPM $\phi$ s from untreated mice (not shown). LPM $\phi$ s from IL-10R $\alpha^{\mathrm{f} / \mathrm{fl}}$ mice with colitis stained positively for ROS (Figure 6a). However, IL- $10 \mathrm{R} \alpha^{\mathrm{Mdel}} \mathrm{LPM} \phi$ s displayed a significant increase in this $\left(\mathrm{IL}-10 \mathrm{R} \alpha^{\mathrm{f} / / \mathrm{fl}} \mathrm{MFI}=42.9 \pm 14.1, \quad \mathrm{IL}-10 \mathrm{R} \alpha^{\mathrm{Mdel}} \mathrm{MFI}=134.0 \pm\right.$ 24.4, $P<0.01)$. This increase in ROS was specific for macrophages; LP-derived DCs, B cells, and T cells did not show this difference. Some splenic macrophages showed detectable ROS production; however, quantities were substantially decreased compared with the LP and did not differ between IL-10R $\alpha^{\text {Mdel }}$ and IL-10R $\alpha^{\mathrm{fl} / \mathrm{fl}}$ mice. Therefore, ROS production is markedly and selectively elevated in IL-10R $\alpha^{\text {Mdel }}$ LPM $\phi$ s.

To clarify the role of the increased IL- $10 \mathrm{R} \alpha^{\text {Mdel }}$ ROS, we treated the mice with an ROS scavenger, $\mathrm{N}$-acetyl-L-cysteine (NAC). NAC did not affect the weight loss or colon length in $\mathrm{IL}-10 \mathrm{R} \alpha^{\mathrm{fl} / \mathrm{fl}}$ mice (Figure 6b,c and Supplementary Figure S9). Comparison of treated and untreated mice did show a trend toward a decrease in bleeding scores, but this was not significant (Figure 6f). The limited effect of NAC in WT mice was not unexpected considering the similar previously documented results with $\mathrm{p} 47$ phox deficiency. However, in IL-10R $\alpha^{\text {Mdel }}$ mice, where ROS production is elevated, NAC led to a more substantial attenuation of disease. Maximal weight loss was decreased from $27 \pm 2$ to $21 \pm 2 \%$ (Figure $6 \mathbf{b}, \mathbf{d}, P<0.05$ ). A trend toward decreased bleeding score was seen but, similar to $\mathrm{IL}-10 \mathrm{R} \alpha^{\mathrm{fl} / \mathrm{fl}}$ mice, was not significant (Figure $6 \mathbf{f}$ ). Therefore, antioxidant treatment shows greater effectiveness in IL- $10 \mathrm{R} \alpha^{\mathrm{Mdel}}$ than IL-10R $\alpha^{\mathrm{fl} / \mathrm{fl}}$ mice.

NAC may also protect against ROS, such as peroxynitrites, formed by the reaction of ROS and NO. Therefore, part of its activity may be secondary to its effects on NO-derived species. To determine if NAC actions were still discernible after inhibiting iNOS, we treated mice with both AG and NAC (Figure 6b-e). These demonstrated complementary effects. Dually treated control IL-10R $\alpha^{\mathrm{fl} / \mathrm{fl}}$ mice showed a limited improvement over untreated or NAC-only treated mice (Figure 6b,c). Their bleeding scores were not significantly improved compared with mice treated with NAC alone but were compared with untreated animals (Figure 6f). In contrast, IL-10R $\alpha^{\text {Mdel }}$ mice treated with both inhibitors showed markedly diminished weight loss, with a significant effect compared with NAC treatment by itself (Figure $\mathbf{6 b}, \mathbf{d}, \mathbf{e}$ ). Peak weight loss of treated IL-10R $\alpha^{\text {Mdel }}$ mice did not significantly differ from that of untreated IL-10R $\alpha^{\mathrm{fl} / \mathrm{fl}}$ controls and was only mildly more severe than similarly treated IL-10R $\alpha^{\mathrm{f} / \mathrm{fl}}$ mice. The bleeding scores of treated IL-10R $\alpha^{\text {Mdel }}$ mice did not significantly differ from untreated IL-10R $\alpha^{\mathrm{fl} / \mathrm{fl}}$ mice, although they did remain elevated compared with NAC or NAC- and AG-treated IL-10R $\alpha^{\mathrm{fl} / \mathrm{fl}}$ controls (Figure 6f). Therefore, dual inhibition of the ROS and iNOS pathways substantially 


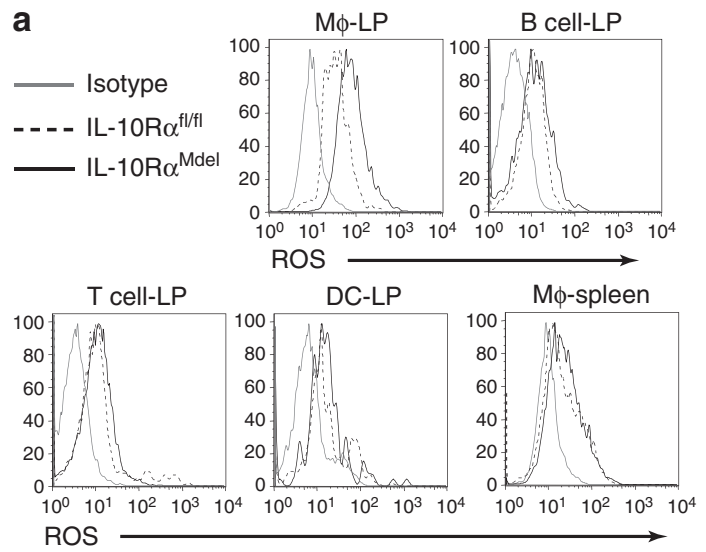

b

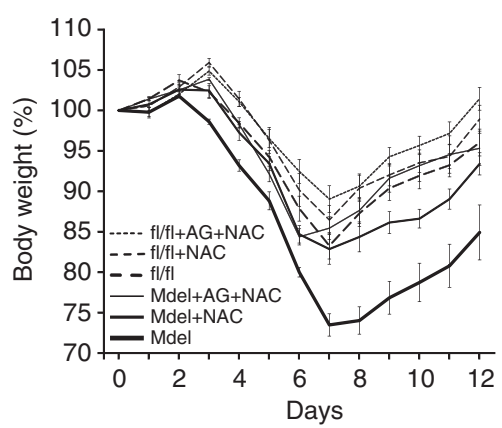

C
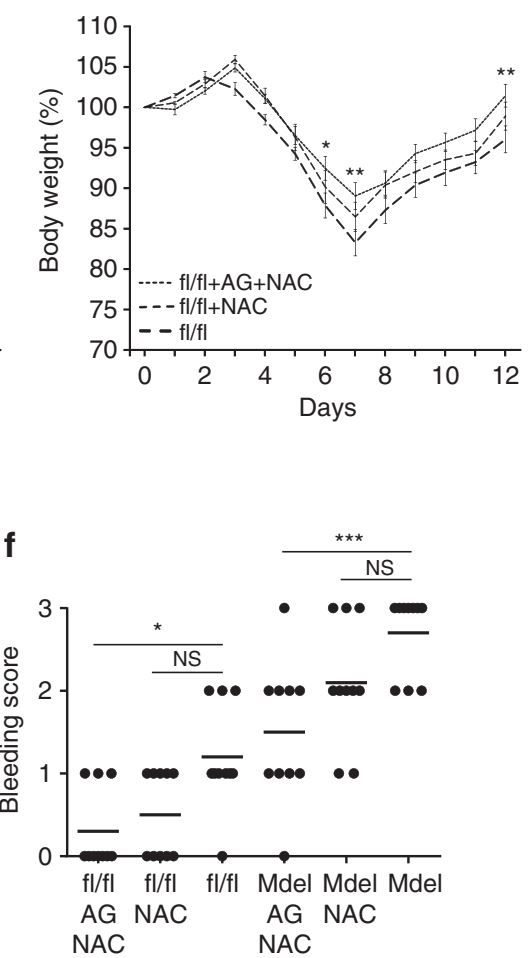

Figure 6 Role of reactive oxygen species (ROS) in colitis exacerbation. (a) On day 7 after colitis induction, gated T cells, B cells, dendritic cells (DCs), and macrophages were analyzed by flow cytometry in the lamina propria (LP) or spleen as indicated. Gray line, isotype control staining of interleukin (IL)-10R $\alpha^{\text {Mdel }}$ cells; dashed black line, IL-10R $\alpha^{\text {fl/fl }}$ cells; solid black line, IL-10R $\alpha^{\text {Mdel }}$ cells. (b-e) Colitis was induced with $3 \%$ dextran sodium sulfate (DSS) in IL-10R $\alpha^{\text {Mdel }}$ and IL-10R $\alpha^{\text {fl/fl }}$ mice that were treated with $N$-acetyl-L-cysteine (NAC) with or without aminoguanidine hydrochloride (AG) or saline intraperitoneally (i.p.). Mean \pm 1 s.e.m. weight change is measured. Plots $b$-e show results for all cohorts, IL-10R $\alpha^{\text {fl/fl }} \operatorname{cohorts,~IL-10R~} \alpha^{\mathrm{Mdel}} \operatorname{cohorts,}$ and a comparison of NAC + AG-treated IL-10R $\alpha$ Mdel with untreated or NAC + AG-treated IL-10R $\alpha^{\mathrm{t} / f \mathrm{fl}}$ cohorts, respectively. ${ }^{\star} P<0.05,{ }^{* *} P<0.01$ comparison of $A G+N A C$ vs. untreated mice in panels $\mathbf{c}$ and d, and $A G+N A C$-treated IL-10R $\alpha^{\mathrm{fl} / \mathrm{fl}} \mathrm{vs}$. IL-10R $\alpha^{\mathrm{Mdel}} \mathrm{cohorts}$ in panel e. ${ }^{\circ} P<0.05,{ }^{\circ} P<0.01$ for NAC vs. untreated cohorts in panels $\mathbf{c}$ and d. ${ }^{\triangle} \triangle P<0.01$ for AG + NAC vs. NAC in panel d. (f) Rectal bleeding scores on day 7 after DSS treatment. ${ }^{\star} P<0.05,{ }^{* * *} P<0.001$, NS, not significant. Comparisons are only shown for treated vs. untreated IL-10R $\alpha^{\mathrm{fl} / \mathrm{fl}}$ or IL-10R $\alpha^{\mathrm{Mdel}}$ cohorts and not between IL-10R $\alpha^{\mathrm{fl} / \mathrm{fl}}$ and IL-10R $\alpha^{\mathrm{Mdel}}$ mice. Data are representative of two independent experiments.

alleviates the enhanced disease in IL-10R $\alpha^{\mathrm{Mdel}}$ mice, whereas it more modestly affects disease in IL-10R $\alpha^{\mathrm{fl} / \mathrm{fl}}$ controls.

\section{DISCUSSION}

Intestinal immune inflammatory and regulatory pathways exist in a highly dynamic balance, ensuring that inevitable disruptions in the mucosal barrier are repaired without undo inflammation or the development of a self-perpetuating colitic process. IL-10 plays a critical role in this and governs IBD susceptibility. Macrophages may serve as a specific control point. MyD88 deletion in macrophages or DCs but not intestinal epithelial cells affects spontaneous colitis in IL-10 $0^{-1-}$ mice. ${ }^{29}$ Macrophagespecific deletion of signal transducer and activator of transcription 3, which signals downstream of multiple cytokines including IL- $10,{ }^{30}$ leads to chronic IBD. However, the cell types directly responsible for the protective effects of IL-10 in colitis have not been definitively resolved.

We did not identify a role for nonhematopoietic IL-10R $\alpha$ expression in IL-10-mediated colitis protection. Similarly, IL-10R $\alpha^{\text {Tdel }}$, IL- $10 R \alpha^{\text {Bdel }}$, and IL-10R $\alpha^{\text {DCdel }}$ mice developed DSS colitis with a kinetics and severity identical to WT controls. In contrast, IL-10R $\alpha^{\text {Mdel }}$ mice manifested more severe disease with increased mortality. This was comparable to that of mice wholly deficient in IL-10 or IL-10R $\alpha$. Granulocyte depletion further implicated macrophages as the primary cellular target for IL-10 after mucosal breech with DSS. Histopathologic changes in IL-10R $\alpha^{\mathrm{Mdel}}$ mice were consistent with an increase in disease magnitude compared with WT controls, but not an altered disease quality.

The absence of a T-specific IL-10 effect is notable considering the documented effect of IL-10 in regulatory T-cell maintenance and disease severity in colitis induced by T-cell transfer into $\mathrm{Rag}^{-1-}$ mice. ${ }^{31}$ Similarly, T-cell response to IL-10 has been implicated in the immunoregulation of intestinal inflammation after $\alpha \mathrm{CD} 3$ treatment. ${ }^{32}$ Contrasting with these models, T cells appear to have a limited involvement in DSS colitis, reflecting the acute toxic influence of DSS on the colonic barrier and subsequent innate inflammatory response. Although changes in the T-cell compartment are evident after DSS treatment, this lineage is not essential for the colitis that may be comparably induced in $\mathrm{Rag}^{-1-}$ mice and immunoreplete mice. ${ }^{33,34}$

It is also notable that IL- $10 \mathrm{R} \alpha^{\text {DCdel }}$ mice did not develop exacerbated DSS colitis. Many macrophages identified in the colon during DSS colitis demonstrated a CD11c dim 
immunophenotype (Supplementary Figure S5 online). Furthermore, sorted $\mathrm{CD} 11 \mathrm{c}^{-}$and CD11 $\mathrm{c}^{\mathrm{dim}} \mathrm{LPM} \phi \mathrm{s}$ from IL-10R $\alpha^{\text {Mdel }}$ mice both showed elevated levels of iNOS, TNF $\alpha$, and IL- $1 \beta$ relative to controls (data not shown). This suggests that both $\mathrm{CD} 11 \mathrm{c}^{-}$and $\mathrm{CD} 11 \mathrm{c}^{\mathrm{dim}}$ populations contribute to the increased IL-10R $\alpha^{\mathrm{Mdel}}$ disease severity. The time course for disease in DSS colitis is highly abbreviated. One possible explanation for the lack of a CD11c-Cre effect is that as monocytes enter the colon, mature into $\operatorname{LPM} \phi$, and some upregulate $\mathrm{CD} 11 \mathrm{c}$, there is insufficient time to induce Cre and delete the IL-10R $\alpha$ gene, and for pre-existing expressed IL-10R $\alpha$ protein to be depleted. Further comparisons of the IL-10R $\alpha^{\text {DCdel }}$ and IL-10R $\alpha^{\text {Mdel }}$ mice are, however, warranted to clarify the mechanism(s) underlying the distinct effects of these different Cre transgenes.

LPM $\phi$ s are activated in all commonly studied colitis models, ${ }^{31,35,36}$ and we further assessed how their inability to respond to IL-10 is linked to exacerbated colitis. DSS disrupts the mucosal barrier. Defective epithelial regeneration may aggravate colitis, ${ }^{21}$ although it was not observed here.

IL-10 suppresses macrophage proinflammatory cytokine production, and colitic IL-10R $\alpha^{\text {Mdel }} \mathrm{LPM} \phi$ s produced more IL-1 $\beta$ and TNF- $\alpha$ than controls, although IL-10 itself was unaltered. The T-cell response is not essential to DSS colitis, ${ }^{34}$ and in pilot studies we found no differences in interferon- $\gamma$, IL-17, and IL-23 production by quantitative real-time reversetranscriptase-PCR (data not shown). Although proinflammatory cytokines are broadly elevated in IL-10R $\alpha^{\text {Mdel }}$ colitis, we focused on TNF- $\alpha$ because of its direct cytopathic effects and prominent regulation by IL-10 in macrophage. TNF- $\alpha$ inhibition proved protective in both IL-10R $\alpha^{\text {Mdel }}$ mice and controls, but to a similar extent, and disease in treated IL-10R $\alpha^{\text {Mdel }}$ mice remained more severe than in even untreated IL-10R $\alpha^{\mathrm{f} / \mathrm{fl}}$ controls. Therefore, although TNF- $\alpha$ plays a role in colitis development, it cannot in itself explain the increased IL-10R $\alpha^{\text {Mdel }}$ disease susceptibility.

We did not observe differences in macrophage numbers, phenotype, or segregation into SSC ${ }^{\text {hi }}$ and SSClo LPM $\phi 1$ and LPM $\phi 2$ populations. Similarly, mixed chimeras demonstrated that IL-10R $\alpha^{\text {Mdel }}$ macrophages do not outcompete WT macrophages during colitis development, indicating that IL10 is not affecting cellular localization, migration, or expansion. However, IL-10R $\alpha^{\text {Mdel }}$ macrophages showed markedly elevated $\mathrm{NO}$ and ROS production that are important for clearing bacteria that traverse the disrupted mucosal barrier, ${ }^{37,38}$ although in excess they may also mediate direct tissue damage. ${ }^{26,28,39-41}$

Importantly, inhibition of either NO or ROS led to no or modest effects on colitis severity in WT (IL-10R $\left.\alpha^{\mathrm{fl} / \mathrm{fl}}\right)$ mice. This is consistent with the mixed protective and pathologic functions of these agents. In contrast, colitis was more substantially alleviated by their inhibition in IL-10R $\alpha^{\text {Mdel }}$ mice. Indeed, weight loss in NAC/AG-treated IL-10R $\alpha^{\text {Mdel }}$ was only mildly increased compared with similarly treated WT controls, indicating that inhibition of these pathways converts the more extreme disease in IL-10R $\alpha^{\mathrm{Mdel}}$ mice to one similar to that of WT mice. Limited studies have been performed in DSS colitis to identify immunopathologic mechanisms of ROS and $\mathrm{NO}$, and it will be important in the future to further clarify how IL-10 influences the effects of these molecules. Nevertheless, our results are consistent with a model in which intestinal IL-10 acts to downregulate macrophage NO and ROS production after barrier insult. In the absence of adequate IL-10 signaling, damage produced by these mediators amplifies the toxic insult from DSS treatment and aggravates disease. The limited effect of NO and ROS inhibition in WT mice implies that IL-10 normally reduces these compounds to a level where their direct toxic actions are roughly balanced by their protective functions.

In summary, we demonstrate an indispensable and dominant role for macrophage IL-10 responsiveness in the protective effects of IL-10 in colitis development. We further demonstrate that IL-10 does not alter the competitive fitness of macrophages themselves, but rather impairs their effector functions, and most particularly their excessive production of pathologic reactive oxygen and nitrogen species.

\section{METHODS}

Mice. IL-10R $\alpha^{\mathrm{fl} / \mathrm{fl}}$ mice were generated on a C57BL/6 background as described previously ${ }^{18}$ and bred with B6.129P2-Lyzs ${ }^{\operatorname{tm} 1 \text { (cre)Ifo } / J}$ (Lys-cre, The Jackson Laboratories), B6.Cg-Tg(Cd4-Cre)1Cwi/BfluJ (CD4-cre, gift of H. Chi, St Jude Children's Research Hospital, Memphis, TN), CD11c-cre (gift of H. Chi), B6.129P2-CD19 ${ }^{\mathrm{tm} 1(\mathrm{cre}) \mathrm{Cgn}} / \mathrm{J}$ (CD19-cre, The Jackson Laboratories, Bar Harbor, ME), and B6.C-Tg(CMV-cre)1Cgn/J (CMV-cre, The Jackson Laboratories). B6.129P2-IL-10 $0^{\mathrm{tm} 1 \mathrm{Cgn}} / \mathrm{J}$ mice were obtained from The Jackson Laboratories. Mice were maintained under specific pathogen-free conditions negative for detectable Helicobacter spp. Experimental protocols were approved by the St Jude Animal Care and Use Committee.

Induction of colitis and clinical scoring. DSS (molecular weight 40,000; ICN Biomedicals, Santa Ana, CA) was administered ad libitum in distilled water at $3 \%$ concentration or as indicated for 5 days followed by normal drinking water. For inhibition experiments, NAC $\left(100 \mathrm{mg} \mathrm{kg}^{-1}\right.$, Sigma, St Louis, MO), AG (100 $\mathrm{mg} \mathrm{kg}^{-1}$, Calbiochem, Darmstadt, Germany), or BEC (20 mg kg-1, Sigma) was administered intraperitoneally. Neutrophils were depleted using anti-Ly6G monoclonal Ab 1 A8 (Bio X Cell, West Lebanon, NH). A total of $1 \mathrm{mg}$ Ab per mouse was administered intraperitoneally 1 day before DSS treatment. Depletion was confirmed by flow cytometry. Body weight and gross blood were analyzed on a daily basis. ${ }^{42}$ Bleeding scores were: 0 , hemoccult negative (Beckman Coulter, Brea, CA); 1, hemoccult positive; 2, blood traces in stool; and 3, gross rectal bleeding.

Histology. Colons (day 7) were stained with hematoxylin and eosin. Three independent sections were assessed per mouse by a blinded reviewer. Inflammation scoring was as follows: 0, no or occasional inflammatory cells in the LP; 1 , increased LP inflammatory cells; 2 , confluence of inflammatory cells extending into the submucosa; and 3 , transmural infiltrate extension of the infiltrate. Ulceration scoring was as follows: 0 , no ulceration; 1 , mild (1-2 ulcers per 40 crypts analyzed); 2, moderate (3-4 ulcers); and 3, severe ( $>4$ ulcers). Hyperplasia scoring was as follows: 0 , normal; 1 , crypts up to twice the normal thickness of normal epithelium; 2, crypts $>2$ times normal thickness, hyperchromatic epithelium; reduced goblet cells, scattered arborization; and 3 , crypts $>4$ times normal thickness, marked hyperchromasia, few to no goblet cells, high mitotic index, frequent arborization. Disease area scoring was as follows: $0,0-5 \%$ involvement; $1,5-30 \% ; 2,30-70 \%$; and $3,>70 \%$. Total score is the sum of individual scores.

Cytokine levels. Frozen colon samples were homogenized in ice-cold phosphate-buffered saline containing $1 \%$ NP-40 and complete 
protease inhibitor cocktail (Roche, Basel, Switzerland). Cytokines and chemokines in samples were directly measured by Luminex (Bio-Rad, Hercules, CA) or ELISA (R\&D Systems, Minneapolis, MN).

LP cell isolation. LP cells were isolated using a modification of a previously described protocol. ${ }^{43}$ Briefly, colon segments were twice vigorously shaken in medium with $1 \mathrm{~mm}$ EDTA (Sigma-Aldrich, St Louis, MO) for $20 \mathrm{~min}$ at $37^{\circ} \mathrm{C}$, and suspended cells collected and filtered through a cell strainer. Tissue was further minced and incubated at $37^{\circ} \mathrm{C}$ for $1 \mathrm{~h}$ in medium with $1 \mathrm{~mm}$ collagenase type IV (Sigma-Aldrich) and $40 \mathrm{U} \mathrm{ml}^{-1}$ DNase I (Roche) with agitation. Cells were filtered, washed, and isolated over a Percoll step gradient (GE Healthcare, Pittsburgh, PA).

Bone marrow chimeras. Chimeras were produced as previously described. ${ }^{44}$ Briefly, $\sim 5 \times 10^{6}$ donor bone marrow cells were transplanted into lethally irradiated C57BL/6J recipients. Reconstitution was verified after 4 weeks by staining peripheral blood for the transplanted cells. Colitis was induced at 8 weeks.

Intestinal permeability. Epithelial barrier permeability was assessed using FITC-labeled dextran as described previously. ${ }^{21}$ Briefly, mice were gavaged with FITC-dextran (Sigma-Aldrich, $1 \mathrm{~g} \mathrm{~kg}^{-1}$ ) on day 7 . After $6 \mathrm{~h}$, blood was collected and the plasma FITC-dextran quantified by fluorescence spectrophotometry.

Epithelial cell proliferation. Proliferating intestinal epithelial cells were quantified as described previously. ${ }^{45}$ Briefly, 5-bromodeoxyuridine $\left(20 \mathrm{mg} \mathrm{kg}^{-1}\right)$ was administered intraperitoneally to mice with colitis (day 7) or untreated mice. After $2 \mathrm{~h}$, colons were removed and cells incorporating 5-bromodeoxyuridine quantified by immunohistochemistry. The average number of 5-bromodeoxyuridine-positive epithelial cells per intact and well-oriented crypt was determined (minimum of 50 crypts assessed per mouse).

Cytokine PCR. Total RNA was isolated from sorted LPM $\phi$ s using the RNeasy mini kit (Qiagen, Venlo, The Netherlands), and complementary DNA synthesized using superscript III and oligo (dT) primers (Invitrogen, Carlsbad, CA). Expression levels were normalized to HPRT $(\Delta \mathrm{Ct})$ and compared with littermate controls using the $\Delta \Delta \mathrm{Ct}$ method. ${ }^{46}$ Primer sequences are: transforming growth factor- $\beta$ : forward F, 5'-CACAGTACAGCAAGGTCCTTGC-3'; reverse R, 5'-AGTAGACGATGGGCAGTGGCT-3'; IL-12p35: F, 5' -ATGACCC TGTGCCTTGGTAG-3'; R, 5'-GATTCTGAAGTGCTGCGTTG-3'; IL-23p19: F, 5'-AGCGGGACATATGAATCTACTAAGAGA-3'; R, 5'-GTCCTAGTAGGGAGGTGTGAAGTT-3'; IL-12p40: F, 5'-GAC CATCACTGTCAAAGAGTTTCTAGAT- ${ }^{\prime} ;$ R, $5^{\prime}$-AGGAAAGTCTT GTTTTTGAAATTTTTTAA-3'; IL-10: F， 5'-GTGAAAATAAGAG CAAGGCAGTG- $3^{\prime} ; \quad$ R, $5^{\prime}$-ATTCATGGCCTTGTAGACACC- $3^{\prime}$; TNF- $\alpha$ : F, $5^{\prime}$-AATGGCCTCCCTCTCATCAGT- $3^{\prime}$; R, $5^{\prime}$-CTACAGG CTTGTCACTCGAA-3'; iNOS: F, 5' -TGACGGCAAACATGACTTC AG-3'; R, 5'-GCCATCGGGCATCTGGTA-3'; IL-6: F, 5' -TATGAA GTTCCTCTCTGCAAGAGA-3'; R, 5'-TAGGGAAGGCCGTGGT

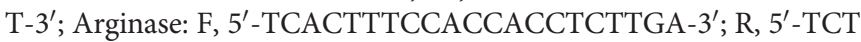
CCACCGCCTCACGACTC-3'; HPRT: F, 5'-GACCGGTCCCGTCA TGC-3'; R, 5' -TCATAACCTGGTTCATCATCGC-3'.

Flow cytometry. LPM $\phi$ s were stained with monoclonal Abs against mouse F4/80, CD11b, CD11c, CD40, CD80, CD86, major histocompatibility complex class II, CD103, Toll-like receptor 2, CD45.1, CD45.2, Ly6G, and Siglec-F, or with isotype-matched control Abs (BD Pharmingen, Franklin Lakes, NJ or eBiosciences, San Diego, CA), and analyzed using a FACSCalibur or LSRII flow cytometer with Cell Quest (BD Biosciences, Franklin Lakes, NJ) or Flowjo (TreeStar, Ashland, OR) software.

NOS activity. LP cells were isolated, homogenized in $200 \mu \mathrm{l}$ lysis buffer $(80 \mathrm{~mm}$ sodium phosphate, $\mathrm{pH} 6$, containing $0.5 \%$ hexadecyltrimethyl ammonium bromide, Sigma-Aldrich) for $60 \mathrm{~s}$, centrifuged at $14,000 \times g$ for $15 \mathrm{~min}$, and supernatant protein determined by Bradford assay (Bio-Rad). NOS activity was measured by an ultrasensitive colorimetric assay (Oxford Biomedical Research, Rochester Hills, MI, cat. no. NB 78) as per the manufacturer's instructions.

ROS staining. LP cells were stained for surface markers, incubated for 30 min at $37^{\circ} \mathrm{C}$ with $10 \mu \mathrm{M}$ CM-H2DCFDA (Invitrogen) and analyzed by flow cytometry. ${ }^{47}$

Statistics. Statistics were calculated using Prism5 (GraphPad Software, San Diego, CA). Group comparisons were by Student's $t$-test or, when multiple cohorts were present, analysis of variance with Bonferroni correction. A $P<0.05$ was considered significant.

SUPPLEMENTARY MATERIAL is linked to the online version of the paper at http://www.nature.com/mi

\section{ACKNOWLEDGMENTS}

This study was supported by the National Institutes of Health grant R01 Al056153 and R56 Al006600 (to TLG) and the American Lebanese Syrian Associated Charities (ALSAC)/St Jude Children's Research Hospital (to all authors).

\section{DISCLOSURE}

The authors declared no conflict of interest.

c) 2014 Society for Mucosal Immunology

\section{REFERENCES}

1. Arai, Y., Takanashi, H., Kitagawa, H. \& Okayasu, I. Involvement of interleukin-1 in the development of ulcerative colitis induced by dextran sulfate sodium in mice. Cytokine 10, 890-896 (1998).

2. Atreya, R. et al. Blockade of interleukin 6 trans signaling suppresses T-cell resistance against apoptosis in chronic intestinal inflammation: evidence in Crohn disease and experimental colitis in vivo. Nat. Med. 6 , 583-588 (2000).

3. Simpson, S.J. et al. T cell-mediated pathology in two models of experimental colitis depends predominantly on the interleukin 12/Signal transducer and activator of transcription (Stat)-4 pathway, but is not conditional on interferon gamma expression by T cells. J. Exp. Med. 187, 1225-1234 (1998).

4. Yang, X.O. et al. Regulation of inflammatory responses by IL-17F. J. Exp. Med. 205, 1063-1075 (2008).

5. Kojouharoff, G. et al. Neutralization of tumour necrosis factor (TNF) but not of IL-1 reduces inflammation in chronic dextran sulphate sodium-induced colitis in mice. Clin. Exp. Immunol. 107, 353-358 (1997).

6. Maloy, K.J. \& Powrie, F. Intestinal homeostasis and its breakdown in inflammatory bowel disease. Nature 474, 298-306 (2011).

7. Glocker, E.O., Kotlarz, D., Klein, C., Shah, N. \& Grimbacher, B. IL-10 and IL-10 receptor defects in humans. Ann. NY Acad. Sci. 1246, 102-107 (2011).

8. Glocker, E.O. et al. Inflammatory bowel disease and mutations affecting the interleukin-10 receptor. N. Engl. J. Med. 361, 2033-2045 (2009).

9. Kuhn, R., Lohler, J., Rennick, D., Rajewsky, K. \& Muller, W. Interleukin10-deficient mice develop chronic enterocolitis. Cell 75, 263-274 (1993).

10. Spencer, S.D. et al. The orphan receptor CRF2-4 is an essential subunit of the interleukin 10 receptor. J. Exp. Med. 187, 571-578 (1998).

11. Mizoguchi, A., Mizoguchi, E., Takedatsu, H., Blumberg, R.S. \& Bhan, A.K. Chronic intestinal inflammatory condition generates IL-10-producing regulatory B cell subset characterized by CD1d upregulation. Immunity 16, 219-230 (2002).

12. Powrie, F. et al. Inhibition of Th1 responses prevents inflammatory bowel disease in SCID mice reconstituted with CD45RBhi CD4 $+\mathrm{T}$ cells. Immunity 1, 553-562 (1994).

13. Tomoyose, M., Mitsuyama, K., Ishida, H., Toyonaga, A. \& Tanikawa, K. Role of interleukin-10 in a murine model of dextran sulfate sodium-induced colitis. Scand. J. Gastroenterol. 33, 435-440 (1998).

14. Ouyang, W., Rutz, S., Crellin, N.K., Valdez, P.A. \& Hymowitz, S.G. Regulation and functions of the $\mathrm{LL}-10$ family of cytokines in inflammation and disease. Annu. Rev. Immunol. 29, 71-109 (2011). 
15. Moore, K.W., de Waal Malefyt, R., Coffman, R.L. \& O'Garra, A. Interleukin10 and the interleukin-10 receptor. Annu. Rev. Immunol. 19, 683-765 (2001).

16. Mosser, D.M. \& Zhang, X. Interleukin-10: new perspectives on an old cytokine. Immunol. Rev. 226, 205-218 (2008).

17. Saraiva, M. \& O'Garra, A. The regulation of IL-10 production by immune cells. Nat. Rev. Immunol. 10, 170-181 (2010).

18. Liu, X. et al. The T cell response to IL-10 alters cellular dynamics and paradoxically promotes central nervous system autoimmunity. J. Immunol. 189, 669-678 (2011).

19. Clausen, B.E., Burkhardt, C., Reith, W., Renkawitz, R. \& Forster, I. Conditional gene targeting in macrophages and granulocytes using LysMcre mice. Transgenic Res. 8, 265-277 (1999).

20. Daley, J.M., Thomay, A.A., Connolly, M.D., Reichner, J.S. \& Albina, J.E. Use of Ly6G-specific monoclonal antibody to deplete neutrophils in mice. J. Leukoc. Biol. 83, 64-70 (2008).

21. Zaki, M.H. et al. The NLRP3 inflammasome protects against loss of epithelial integrity and mortality during experimental colitis. Immunity $\mathbf{3 2}$ 379-391 (2010).

22. Takada, Y. et al. Monocyte chemoattractant protein-1 contributes to gut homeostasis and intestinal inflammation by composition of IL-10producing regulatory macrophage subset. J. Immunol. 184, 2671-2676 (2010).

23. Rivollier, A., He, J., Kole, A., Valatas, V. \& Kelsall, B.L. Inflammation switches the differentiation program of Ly6Chi monocytes from antiinflammatory macrophages to inflammatory dendritic cells in the colon. J. Exp. Med. 209, 139-155 (2012).

24. Ito, R. et al. Involvement of IL-17A in the pathogenesis of DSS-induced colitis in mice. Biochem. Biophys. Res. Commun. 377, 12-16 (2008).

25. Dharmani, P., Leung, P. \& Chadee, K. Tumor necrosis factor-alpha and Muc2 mucin play major roles in disease onset and progression in dextran sodium sulphate-induced colitis. PLoS One 6, e25058 (2011).

26. Cross, R.K. \& Wilson, K.T. Nitric oxide in inflammatory bowel disease. Inflamm. Bowel Dis. 9, 179-189 (2003).

27. Laroux, F.S., Romero, X., Wetzler, L., Engel, P. \& Terhorst, C. Cutting edge: MyD88 controls phagocyte NADPH oxidase function and killing of gramnegative bacteria. J. Immunol. 175, 5596-5600 (2005).

28. Krieglstein, C.F. et al. Regulation of murine intestinal inflammation by reactive metabolites of oxygen and nitrogen: divergent roles of superoxide and nitric oxide. J. Exp. Med. 194, 1207-1218 (2001).

29. Hoshi, N. et al. MyD88 signalling in colonic mononuclear phagocytes drives colitis in IL-10-deficient mice. Nat. Commun. 3, 1120 (2012).

30. Takeda, K. et al. Enhanced Th1 activity and development of chronic enterocolitis in mice devoid of Stat3 in macrophages and neutrophils. Immunity 10, 39-49 (1999).
31. Murai, M. et al. Interleukin 10 acts on regulatory $T$ cells to maintain expression of the transcription factor Foxp3 and suppressive function in mice with colitis. Nat. Immunol. 10, 1178-1184 (2009).

32. Huber, S. et al. Th17 cells express interleukin-10 receptor and are controlled by Foxp3(-) and Foxp3 + regulatory CD4 + T cells in an interleukin-10-dependent manner. Immunity 34, 554-565 (2011).

33. Dieleman, L.A. et al. Dextran sulfate sodium-induced colitis occurs in severe combined immunodeficient mice. Gastroenterology 107, 1643-1652 (1994).

34. Axelsson, L.G., Landstrom, E., Goldschmidt, T.J., Gronberg, A. \& Bylund-Fellenius, A.C. Dextran sulfate sodium (DSS) induced experimental colitis in immunodeficient mice: effects in CD4(+) -cell depleted, athymic and NK-cell depleted SCID mice. Inflamm. Res. 45, 181-191 (1996).

35. Waddell, A. etal. Colonic eosinophilic inflammation in experimental colitis is mediated by Ly6C(high) CCR2 $(+)$ inflammatory monocyte/macrophagederived CCL11. J. Immunol. 186, 5993-6003 (2011).

36. Mahida, Y.R. The key role of macrophages in the immunopathogenesis of inflammatory bowel disease. Inflamm. Bowel Dis. 6, 21-33 (2000).

37. Benoit, M., Desnues, B. \& Mege, J.L. Macrophage polarization in bacterial infections. J. Immunol. 181, 3733-3739 (2008).

38. Xie, Q.W. et al. Cloning and characterization of inducible nitric oxide synthase from mouse macrophages. Science 256, 225-228 (1992).

39. McCafferty, D.M., Mudgett, J.S., Swain, M.G. \& Kubes, P. Inducible nitric oxide synthase plays a critical role in resolving intestinal inflammation. Gastroenterology 112, 1022-1027 (1997).

40. Hokari, R. et al. Reduced sensitivity of inducible nitric oxide synthasedeficient mice to chronic colitis. Free Radic. Biol. Med. 31, 153-163 (2001).

41. Kolios, G., Valatas, V. \& Ward, S.G. Nitric oxide in inflammatory bowel disease: a universal messenger in an unsolved puzzle. Immunology 113, 427-437 (2004).

42. Wirtz, S., Neufert, C., Weigmann, B. \& Neurath, M.F. Chemically induced mouse models of intestinal inflammation. Nat. Protoc. 2, 541-546 (2007).

43. Medina-Contreras, O. et al. CX3CR1 regulates intestinal macrophage homeostasis, bacterial translocation, and colitogenic Th17 responses in mice. J. Clin. Invest. 121, 4787-4795 (2011).

44. Garg, P. et al. Selective ablation of matrix metalloproteinase-2 exacerbates experimental colitis: contrasting role of gelatinases in the pathogenesis of colitis. J. Immunol. 177, 4103-4112 (2006).

45. Tsuchiya, T. et al. Role of gamma delta Tcells in the inflammatory response of experimental colitis mice. J. Immunol. 171, 5507-5513 (2003).

46. Kullberg, M.C. et al. IL-23 plays a key role in Helicobacter hepaticusinduced T cell-dependent colitis. J. Exp. Med. 203, 2485-2494 (2006).

47. Yang, K., Neale, G., Green, D.R., He, W. \& Chi, H. The tumor suppressor Tsc1 enforces quiescence of naive Tcells to promote immune homeostasis and function. Nat. Immunol. 12, 888-897 (2011). 